\title{
Uso de carro plataforma para traslado de repuestos de maquinaria pesada, como ayuda ergonómica en bodegueros de la empresa CRCC
}

Use of a platform trolley to transfer spare parts for heavy machinery, as an ergonomic aid in winemakers of the company CRCC

Dalton Fabián Herrera Samaniego. ${ }^{1}$, Manolo Alexander Córdova Suárez. ${ }^{2}$, René Gustavo Herrera Samaniego. ${ }^{3} \&$ María Fernanda Cuenca Cajamarca. ${ }^{4}$

Recibido: 14-07-2021 / Revisado: 28-07-2021 /Aceptado: 18-08-2021/ Publicado: 05-09-2021

DOI: $\underline{\text { https://doi.org/10.33262/anatomiadigital.v4i3.1.1873 }}$

\begin{abstract}
.
Resumen.

Introduction. At present, a progressive Introducción. En la actualidad se ha increase in musculoskeletal disorders has been noted in people who work in copper mines, especially winemakers who lift and transfer loads of materials, heavy equipment, and spare parts. Objective. This research analyzed the use of a platform cart as an ergonomic aid, for the notado un aumento progresivo de afecciones osteomusculares en las personas que trabajan en las minas de cobre, en especial los bodegueros que hacen levantamiento y traslado de carga de materiales, equipo pesado, repuestos. Objetivo. Se analizó el uso de carro

1 Regional Autonomous University of Los Andes, Postgraduate, Ambato, Ecuador, pg.daltonfhs@uniandes.edu.ec, (D) https://orcid.org/0000-0002-4294-3854

${ }_{2}$ National University of Chimborazo, Faculty of Engineering, Industrial Engineering Degree, Riobamba Ecuador, manolo.cordova@unach.edu.ec, (D) https://orcid.org/0000-0001-6786-7926

3 International University of Ecuador, Postgraduate, Quito, Ecuador, reherrerasa@uide.edu.ec, https://orcid.org/0000-0002-8624-3913

4 Regional Autonomous University of Los Andes, Postgraduate, Ambato, Ecuador, pg.mariafcc53@uniandes.edu.ec, (D) https://orcid.org/0000-0002-4294-3854
\end{abstract}


transfer of materials in the warehouse of the company CRCC, using the Snook and Ciriello table, which is based on the ISO 11228 standard. Methodology. This work is prospective, correlational, qualitative-quantitative, since an instrument will be implemented to improve the job of a winemaker. First, the maximum acceptable weight was determined under normal working conditions, considering the worker's posture and the characteristics of the load lifting of the CRCC company cellar position, using the Snook and Ciriello tables that collect the maximum acceptable weights recommended for the load handling, with sustained ascents and descents and the force when pushing or pulling the load. To deepen the study, an evaluation of the lifting and transfer of the load was carried out, after the implementation of a 4-wheel steel platform car with a load capacity of $300 \mathrm{~kg}$, using the KIM-PP Key Indicator push and pull method. Results. From the application of the Snook and Ciriello tables in initial conditions, a value of $12.82 \mathrm{~kg}$ of maximum acceptable weight was obtained, considering: a) manual transport of the load, b) the load is transported by hand, c) $40 \mathrm{~kg}$ of weight medium of the load, d) 10 transports per hour, e) inadequate grip, f) vertical distance from the ground to the hands is $110 \mathrm{~cm}, \mathrm{~g}$ ) distance of transport of the load of $8.5 \mathrm{~m}$. After the implementation of the ergonomic measures, a score of 5 was obtained, which represents a low ergonomic risk level of 1 , taking into account: a) $68 \mathrm{~m}$ push and pull distance, b) use of a platform cart, c) $40 \mathrm{~kg}$ weight of the load, d) speed of movement $<0.8 \mathrm{~m} /$ $\mathrm{s}$, e) trunk slightly inclined forward, f) floor with small unevenness. Conclusion. plataforma como ayuda ergonómica, para el traslado de materiales en la bodega de la empresa CRCC, utilizando la tabla de Snook y Ciriello, que se basa en la norma ISO 11228. Metodología. Este trabajo fue de tipo prospectivo, correlacional, cuali-cuantitativo, ya que se implementó un instrumento que mejore el puesto de bodeguero. Primero se determinó el peso máximo aceptable en condiciones normales de trabajo, considerando la postura del trabajador y las características del levantamiento de carga del puesto de bodeguero de la empresa CRCC, utilizando las tablas de Snook y Ciriello que recogen los pesos máximos aceptables recomendados para la manipulación de cargas, con ascensos y descensos sostenidos y la fuerza cuando se ejerce empuje o arrastre de la carga. Para profundizar el estudio se realizó una evaluación del levantamiento y traslado de la carga, posterior a la implementación de un carro plataforma de acero de 4 ruedas con capacidad de carga de $300 \mathrm{~kg}$, utilizando el método de empuje y tracción Indicador Clave KIM-PP. Resultados. De la aplicación de las tablas de Snook y Ciriello en condiciones iniciales se obtuvo un valor de $12,82 \mathrm{~kg}$ de peso máximo aceptable, considerando: a) transporte manual de la carga, b) la carga es transportada a mano, c) $40 \mathrm{~kg}$ de peso medio de la carga, d) 10 trasportes por hora, e) agarre inadecuado, f) distancia vertical desde el suelo hasta las manos es de $110 \mathrm{~cm}, \mathrm{~g}$ ) distancia de traslado de la carga de $8,5 \mathrm{~m}$. Luego de la implementación de las medidas ergonómicas se obtuvo una puntuación de 5 , que representa un nivel de riesgo ergonómico bajo de 1, tomando en cuenta: a) $68 \mathrm{~m}$ de distancia de empuje y tracción, b) uso de carro plataforma, c) 
The result of the use of the flatbed car helps significantly in the transfer of the cargo of the CRCC company winemakers, determining low risk levels.

Keywords: Grocer, flatbed car, Snook and Ciriello, KIM-PP Key Indicator. 40kg de peso de la carga, d) velocidad de movimiento $<0.8 \mathrm{~m} / \mathrm{s}$, e) tronco ligeramente inclinado hacia adelante, $\mathrm{f}$ ) piso con pequeños desniveles. Conclusión. El resultado del uso del coche plataforma ayuda significativamente en el traslado de la carga de los bodegueros de la empresa $\mathrm{CRCC}$, determinando niveles de riesgo bajo.

Palabras claves: Bodegueros, carro plataforma, Snook y Ciriello, Indicador Clave KIM-PP.

\section{Introducción}

En la actualidad el manejo manual de cargas, es una actividad que se encuentra presente en la mayoría de los puestos de trabajo de las pequeñas y medianas empresas, que se dedican a la producción, especialmente en las minerías (De la Cruz Quispe \& Viza Ticona, 2017), en las cuales, se ha notado el aumento progresivo de algunos problemas de salud, en especial se ve trastornos musculoesqueléticos, ya que los bodegueros realizan manejo y traslado de cargas, las cuales superan los $25 \mathrm{~kg}$ recomendados, dando como resultado cuantiosas pérdidas a la empresa ya que genera ausentismo laboral (Gerena Rojas \& Orjuela Aranda, 2016).

En la mayoría de los países subdesarrollados, la salud ocupacional está tomando vigor dentro de las empresas, siendo la ergonomía una alternativa muy viable para atenuar accidentes y enfermedades profesionales (Carita \& Ynés, 2018). Por otro lado, el uso de ayudas ergonómicas mecánicas disminuye el riesgo ergonómico y por tanto el índice de afecciones osteomusculares en los bodegueros (Monteza López, 2017), que realizan traslados manuales de materiales y repuestos de maquinaria pesada en las actividades de trabajo de las minas de extracción de cobre de la empresa CRCC.

Es importante mencionar que, al realizar traslado de cargas a distancias cortas, no solo genera lesiones leves a corto o largo plazo (Galarzo, García, Merino, Martínez, \& Collado, 2013), sino que intervienen algunos factores como, pisos a desnivel, deslizantes, y obstáculos que pueden originar afecciones más graves como accidentes laborales (Barios García, Durán Palomino, \& Cuasquer Coral, 2013).

Para evaluar el levantamiento y traslado de cargas existen algunos métodos ergonómicos, dentro de los cuales una de las mejores opciones es la tabla de Snook y Ciriello (Ruiz, 2015), ya que permite identificar el peso máximo aceptable promedio de carga, el cual se basa en la norma ISO 11228 (Occhipinti \& Colombini, 2021), y está constituido por varios elementos, como son: a) transporte manual de la carga, b) la carga es transportada 
a mano, c) peso medio de la carga, d) la frecuencia e) agarre inadecuado, f) la carga se manipula alejada del cuerpo, g) distancia vertical desde el suelo hasta las manos, h) distancia de recorrido (Quiroz-Rubiano, 2019).

La utilización de un carro plataforma como ayuda ergonómica, en el puesto de trabajo, se ha evidenciado que disminuye considerablemente el riesgo ergonómico de los bodegueros, y por lo tanto las afecciones osteomusculares (Bellido Guillen, 2015). Para evaluar dicha ayuda, el método más recomendado, es el Indicador Clave KIM-PP (Pinto, Córdova, \& Quinceno, 2012), el cual consiste en la aplicación de ecuaciones de forma sistemática, que incluyen; a) distancia de empuje y tracción, b) uso de carro plataforma, c) peso de la carga, d) velocidad de movimiento, e) posición del tronco, f) condiciones del piso (Celedon, 2018).

Este trabajo será una guía para la implementación de medidas mecánicas correctivas, con el fin de mitigar las lesiones osteomusculares que se generan en el puesto de bodeguero.

\section{Metodología}

La modalidad investigativa empleada para la presente investigación es de tipo prospectivo, correlacional, cuali-cuantitativa (Díaz-Narváez \& Núñez, 2016), ya que se implementará un instrumento que ayude a disminuir el riesgo ergonómico del puesto de trabajo (Tapia Murillo, 2012), para la evaluación se utilizará la tabla de Snook y Ciriello y posterior a la implementación del carro plataforma manual (Asensio, Diego, \& Alcaide Marzal, 2008), se realizará una nueva valoración, utilizando el método de empuje y tracción Indicador Clave KIM-PP.

\section{Evaluación del Nivel ergonómico con la Tabla de Snook y Ciriello}

Se utilizó la Tabla de Snook y Ciriello, para estimar los elementos que intervienen en el resultado de levantamiento y desplazamiento de cargas (Ergonautas, 2015). Se inició tomando los datos generales del puesto de trabajo; la descripción de la empresa como tal, el área, sección y el puesto de trabajo, de una forma exhaustiva, identificando todos los elementos que lo conforman. Luego se tomó datos del trabajador (bodeguero), como; nombres, sexo, edad, antigüedad en el puesto de trabajo, tiempo que ocupa en el puesto por jornada y duración de su jornada laboral. Para la evaluación se consideró la postura del trabajador, que consiste en el transporte de carga, la cual fue representada por una ilustración (Asensio Cuesta, Bastante Ceca, \& Diego Más, 2012), posteriormente se seleccionó las características de transporte de carga, en la cual se registró, el peso medio de la carga que va de 0 - 60kg, el sexo tanto hombre como mujer, el porcentaje de población a proteger que corresponde a 90, 75, 50, 25 y 10, la frecuencia considerando si son transportes por minuto o por hora. Así mismo se investiga si la carga no permite un agarre adecuado o si se manipula alejada del cuerpo. Finalmente se identificó la distancia vertical desde el suelo hasta las manos que va desde $0-175 \mathrm{~cm}$, y la distancia de recorrido (Ergonautas, 2015). 
Evaluación del nivel ergonómico luego de la implementación del carro plataforma, utilizando el Método de empuje y tracción Indicador Clave KIM-PP

Se realizó una nueva valoración ergonómica, posterior a la implementación de un carro plataforma manual, plegable, de acero con 4 ruedas (Hernández Medina \& Vásquez Ríos, 2020), utilizando el método de empuje y tracción Indicador Clave KIM-PP, que consiste en la aplicación de ecuaciones de forma sistemática, la cual incluyen; a) distancia de empuje y tracción total por día de trabajo menor a $300 \mathrm{~m}$, b) uso de carro plataforma c) peso de la carga menor a $50 \mathrm{~kg}$, d) velocidad de movimiento menor a $0,8 \mathrm{~m} / \mathrm{s}$, e) posición del tronco, ligeramente inclinado hacia adelante, f) condiciones del piso limitadas por pequeños desniveles (Staff, 2015).

\section{Resultados y discusión}

\section{Resultados de la observación de campo}

Tabla 1. Al concluir la evaluación ergonómica con las Tablas de Snook y Ciriello, en el puesto de bodeguero, se obtuvo los siguientes resultados.

Tabla 1. Resultado con las Tablas de Snook y Ciriello

\begin{tabular}{cc}
\hline Elemento & Observación \\
\hline Puesto & Bodeguero \\
Género & Masculino \\
Edad & 36 años \\
Antigüedad del puesto & 5 años \\
Tiempo que ocupa el puesto por jornada & 8 horas \\
Postura del trabajador & Transporte de carga \\
Peso medio de la carga & $40 \mathrm{~kg}$ \\
Población a proteger & $90 \%$ \\
Frecuencia de traslado de carga & 10 transportes/hora \\
Agarre de la carga & No permite un agarre adecuado \\
Distancia recorrida & $110 \mathrm{~cm}$ \\
Peso máximo aceptable & $8,5 \mathrm{~m}$ \\
Ratio & $12,82 \mathrm{~kg}$ \\
\hline
\end{tabular}

Nota: Los datos obtenidos fueron en base a las 8 horas de jornada de trabajo. Es evidente que excede 1, por lo tanto, resulta perjudicial para la salud de los bodegueros.

Tabla 2, muestra los resultados de mejora obtenidos luego de la implementación del carro plataforma, utilizando el Método de empuje y tracción, Indicador Clave KIM-PP. 
Tabla 2. Condiciones del trabajo recomendado

\begin{tabular}{ccc}
\hline ITEM & Elemento & Puntos \\
\hline Distancia de empuje y tracción & $<300 \mathrm{~m}$ & 1 \\
Masa para ser movido & $<50 \mathrm{~kg} /$ uso de carro plataforma & 0,5 \\
Velocidad del movimiento & $<0,8 \mathrm{~m} / \mathrm{s}$ & 1 \\
Posición del tronco & Ligeramente inclinado hacia adelante & 2 \\
Condiciones de trabajo & Restringido (pequeños desniveles) & 2
\end{tabular}

Rango de riesgo $<10$ 5 1

Nota: El resultado obtenido posterior a la implementación de carro plataforma, nos da un rango de riesgo menor a 1. Situación de carga baja.

Figura 1, muestra el carro plataforma empleado para disminuir el riesgo ergonómico, así como la posición que adopta la persona al hacer el traslado de la carga, considerando la distancia de recorrido de $8,5 \mathrm{~m}$.

Figura 1. Condición final del puesto de trabajo.

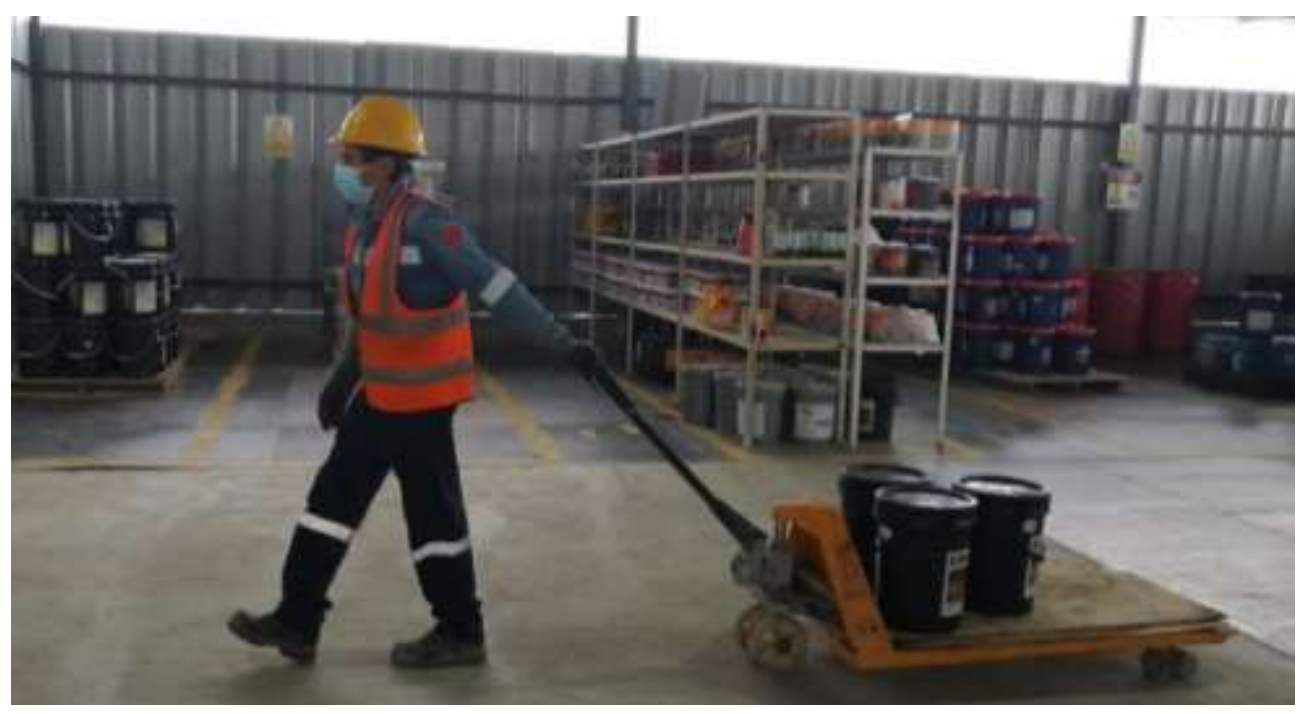

\section{Conclusiones}

El resultado del uso del coche plataforma ayuda significativamente en el traslado de la carga de los bodegueros de la empresa CRCC, determinando niveles de riesgo bajo. Las condiciones de trabajo más recomendadas como bodeguero es un peso máximo de transporte de la carga de $12,82 \mathrm{~kg}$, con una frecuencia de 10 traslados / hora y con una población protegida del $90 \%$.

\section{Referencias bibliográficas}

Asensio Cuesta, S., Bastante Ceca, M. J., \& Diego Más, J. A. (2012). Evaluación ergonómica de puestos de trabajo: Editorial paraninfo. 
Asensio, S., Diego, J., \& Alcaide Marzal, J. (2008). Método para la ayuda al rediseño para la mejora ergonómica de puestos con manipulación manual de cargas.

Barios García, E. Y., Durán Palomino, F., \& Cuasquer Coral, J. A. (2013). Factores ergonómicos que inciden en la ocurrencia de accidentes laborales de origen osteomuscular en trabajadores expuestos a manejos de cargas en la empresa Postobón, sede Pereira años 2008-2012. Universidad Libre de Pereira.

Bellido Guillen, J. G. (2015). Evaluación ergonómica de levantamiento de transporte rutinarios de materiales en obras de construcción civil (caso empresa constructora Valencia SRL 2014 Arequipa).

Carita, V., \& Ynés, V. (2018). Modelo de un sistema de gestión de seguridad y salud ocupacional, según la norma OHSAS 18001, para disminuir accidentes laborales, en una Institución Técnica.

Celedon, A., Stotz, A., Castellucci I. (2018). Guía técnica para la evaluaciòn y control de riesgos asociados al manejo o manipulación manual de cargas. Subsecretaría de prevención social.

De la Cruz Quispe, N. J., \& Viza Ticona, G. Z. (2017). Factores de riesgos ergonómicos que inciden en la salud de los trabajadores del área de producción de la Empresa Andes Yarn SAC, Arequipa-2016.

Díaz-Narváez, V. P., \& Núñez, A. C. J. R. C. d. I. s. (2016). Artículos científicos, tipos de investigación y productividad científica en las Ciencias de la Salud. 14(1), 115-121.

Ergonautas. (2015). Evaluación De La Manipulación Manual De Cargas Mediante Las Tablas De Snook Y Ciriello. Universidad Politécnica De Valencia. Retrieved from Https://Www.Ergonautas.Upv.Es/Metodos/Snook_y_ciriello/Snook-Ayuda.Php

Galarzo, M. C. G., García, A. M. G., Merino, R. G., Martínez, J. M. M., \& Collado, J. M. V. J. R. e. d. s. p. (2013). Exposición a carga física en el trabajo por ocupación: una explotación de los datos en matriz empleo-exposición española (MATEMESP). 87(6), 601-614.

Gerena Rojas, T. Y., \& Orjuela Aranda, A. J. (2016). Revisión documental sobre los desórdenes músculo esqueléticos relacionados con el trabajo en minería.

Hernández Medina, T., \& Vásquez Ríos, A. I. (2020). Propuesta para mejorar el puesto de trabajo en base a la evaluación de riesgos ergonómicos en la empresa Cerámica San Pablo SAC-Yurimaguas.

Monteza López, O. J. (2017). Implementación de un sistema de gestión de seguridad y salud ocupacional en base a la norma OHSAS 18001 en la empresa INDEPAL UCAYALI SA, Neshuya 2016. 
Occhipinti, E., \& Colombini, D. (2021). ISO Technical Report 12295: Application Document for ISO Standards on Manual Handling (ISO 11228-1, ISO 11228-2, and ISO 11228-3) and Evaluation of Static Working Postures (ISO 11226). In Handbook of Standards and Guidelines in Human Factors and Ergonomics (pp. 423-442): CRC Press.

Pinto, R., Córdova, V., \& Quinceno, L. J. C. T. (2012). Ecuaciones de empuje y arrastre ACHS: Relación entre el peso de una carga y la fuerza humana necesaria para moverla. 14(45), 228-232.

Quiroz-Rubiano, M. M. J. C. e. (2019). Laboratorio de Riesgo Ergonómico o Biomecánico. 59-82.

Ruiz, L. R. J. I. N. d. S. e. H. d. T., España. (2015). Manipulación Manual de Cargas. Tablas de Snook y Ciriello. Norma ISO 11228.

Staff, R. J. R. E. d. T. (2015). Método de Indicadores Clave (MIC) para tareas de manipulación de cargas. 5(1), 30-33.

Tapia Murillo, D. N. (2012). Estudio ergonómico comparativo del mobiliario y entorno de trabajo en el personal que labora en la Dirección General Académica, de la Pontificia Universidad Católica del Ecuador, y la relación con la prevalencia de afecciones funcionales en la columna vertebral, año 2009/2011. Quito/PUCE/2012.

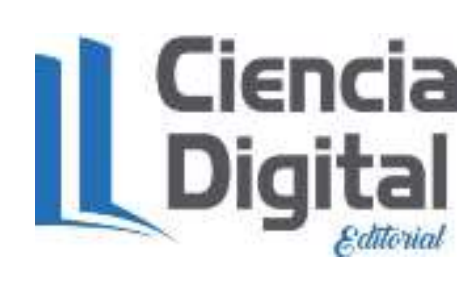




\section{PARA CITAR EL ARTÍCULO INDEXADO.}

Herrera Samaniego, D. F., Córdova Suárez, M. A., Herrera Samaniego, R. G., \& Cuenca Cajamarca, M. F. (2021). Uso de carro plataforma para traslado de repuestos de maquinaria pesada, como ayuda ergonómica en bodegueros de la empresa CRCC. Anatomía Digital, 4(3.1), 98-106. https://doi.org/10.33262/anatomiadigital.v4i3.1.1873

\section{Ciencia \\ LDigital}

El artículo que se publica es de exclusiva responsabilidad de los autores y no necesariamente reflejan el pensamiento de la Revista Anatomía Digital.

El artículo queda en propiedad de la revista y, por tanto, su publicación parcial y/o total en otro medio tiene que ser autorizado por el director de la Revista Anatomía Digital.
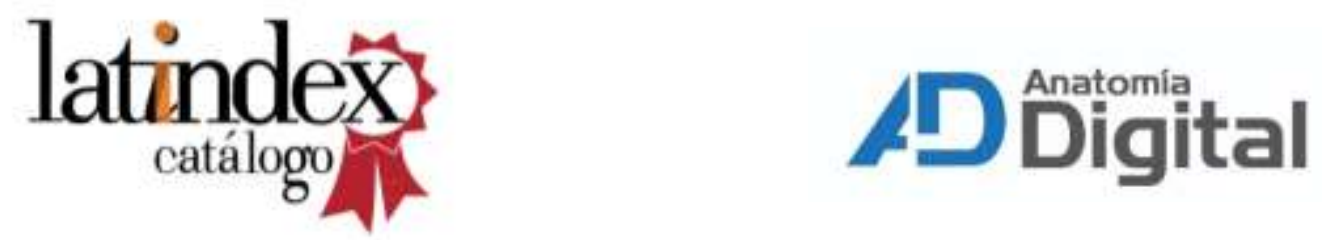